\title{
FIBROSARCOMA OCCURRING IN A CHRONIC BONE SINUS
}

\author{
IV. WAUGH, LONDON, ENGLAND \\ From the Orthopaedic Department, King's College Hospital, London
}

Recent publications by Marks and Turner (1950) and Gillis and Lee (1951) have drawn attention to the occurrence of carcinoma in sinuses of osteomyelitis and as a sequel to war wounds. The case to be described is unusual because the tumour which grew rapidly in relation to a chronic bone sinus of forty-eight years duration proved to be a fungating fibrosarcoma. So far as can be ascertained no similar case has been reported in the British literature.

\section{CASE REPORT}

A man aged sixty-six years was admitted to hospital on account of a rapidly enlarging growth that had appeared three months previously on the outer side of the left thigh at the site of a chronic discharging sinus. In 1903, while in India, he had injured his left knee at

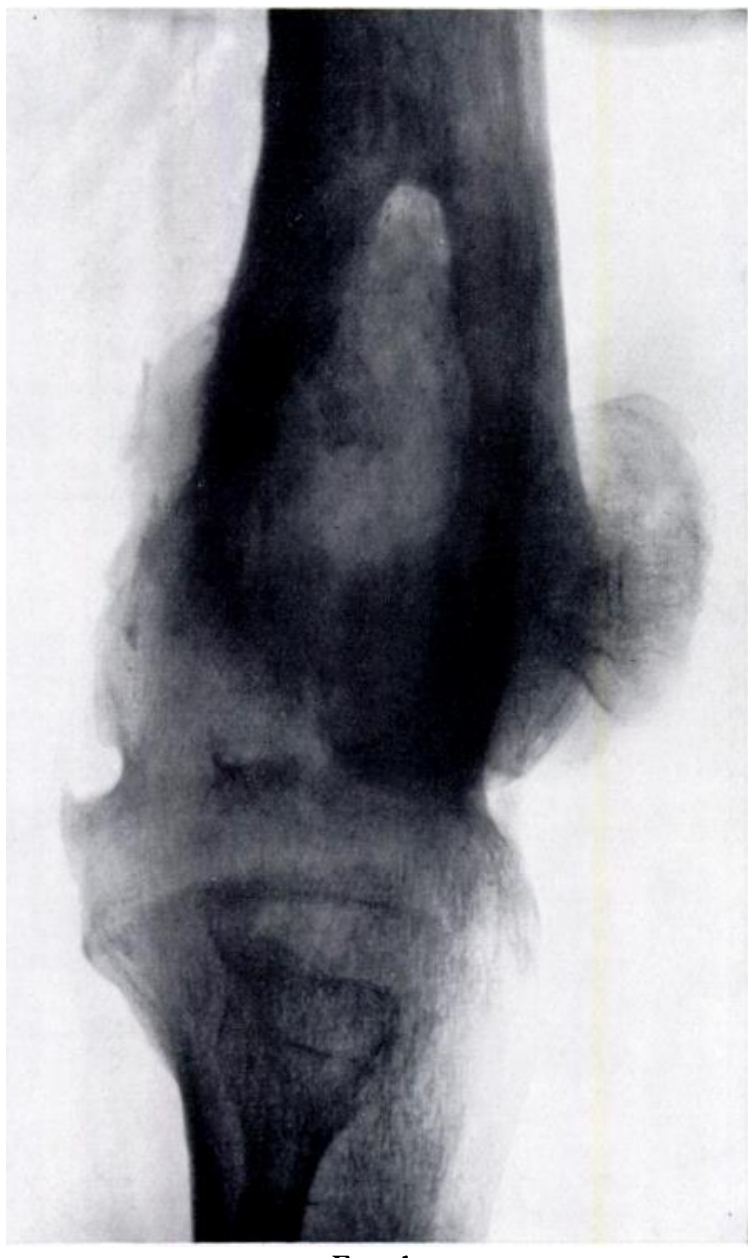

FIG. 1

Radiograph showing the changes in the lower end of the femur. football and the knee had been operated upon. Osteomyelitis developed and he was left with a chronic sinus on the outer surface of the left thigh, and a stiff knee. A further operation was undertaken in 1936 but the sinus continued to discharge. Shortly before admission he had had a rigor and developed a high fever. It was assumed that this was related to an acute flare of his osteomyelitis as no other cause could be found.

On examination, there was a fungating mass four inches in diameter with a base two inches wide at the site of the sinus (Fig. -2). The growth was heavily infected and bled easily but it felt firm. The left knee was ankylosed. The inguinal glands were not palpable.

Radiographs showed a large cavity in the lower end of the femur surrounded by sclerosed bone (Fig. 1). Periosteal new bone formation extended half way up the shaft of the femur. There was bony ankylosis of the knee joint. Radiographs of the chest were normal. Investigations - Blood count showed haemoglobin 56 per cent and red blood corpuscles $\mathbf{2}, \mathbf{4 6 0 , 0 0 0}$ per cubic millimetre. White cells numbered 17,800 per cubic millimetre of which 81 per cent were neutrophils. Blood urea was 56 milligrams per 100 millilitres. 
Biopsy-A wedge-shaped piece of tissue was removed. Histological examination showed a cellular tumour composed mainly of spindle cells with scanty fibre formation. A thick network of reticulin was present and here and there a little collagen had been formed. There were numerous mitoses. The periphery of the growth was necrotic and infiltrated with leucocytes, but the central mass was free from inflammatory cellular infiltration. The conclusion of the pathologist was that the tumour was a growing fibrosarcoma.

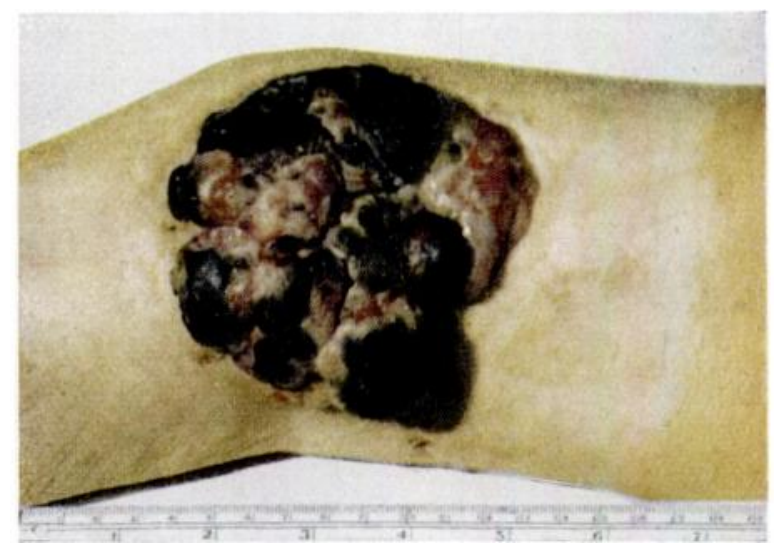

FIG. 2

Photograph showing the gross appearance of the fungating tumour on the outer side of the left thigh.

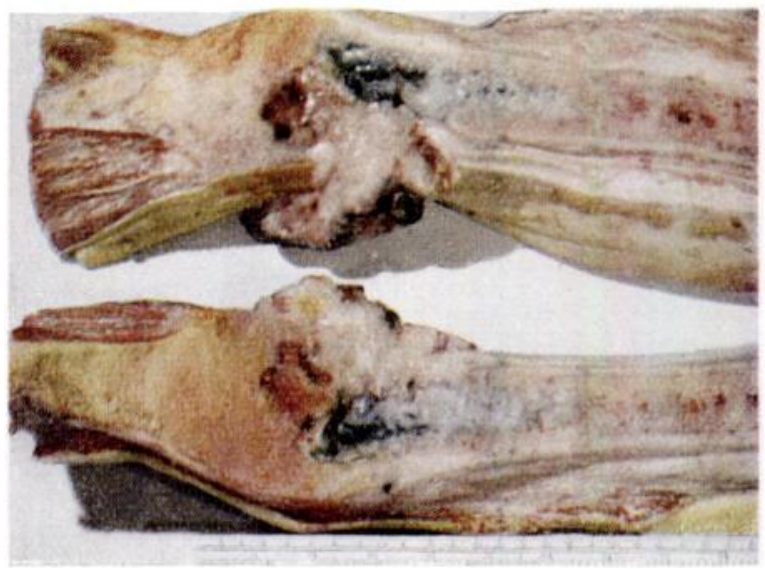

FIG. 3

Photograph of the specimen after it had been divided longitudinally: This shows the extent of the tumour and the underlying abscess.

Treatment-After preliminary blood transfusion and a course of penicillin injections above-knee amputation was carried out.

Pathological examination-The tumour extended through the muscles into the femur (Fig. 3). There was an irregular osteomyelitic abscess in the lower end of the femur. The abscess contained a small sequestrum and was lined by necrotic material except on the medial side, where the growth extended into it through a breach in the cortex. Histological sections

VOL. 34 B, No. 4, NOVEMBER 1952

G2 $(4)$ 
(Figs. 4 and 5) showed the growth to be a sarcoma, the structure being similar to that of the biopsy material. It was composed mainly of spindle-celled tissue although other areas were more anaplastic and there was some necrosis. Spicules of degenerating bone were present around the edges and in one area there were a number of multinucleate giant cells which appeared to be osteoclasts. There was no formation of new bone or its precursors. There was no evidence to suggest that this sarcoma was osteogenic in nature. Presumably it had arisen from granulation tissue lining the sinus leading to the osteomyelitic abscess.

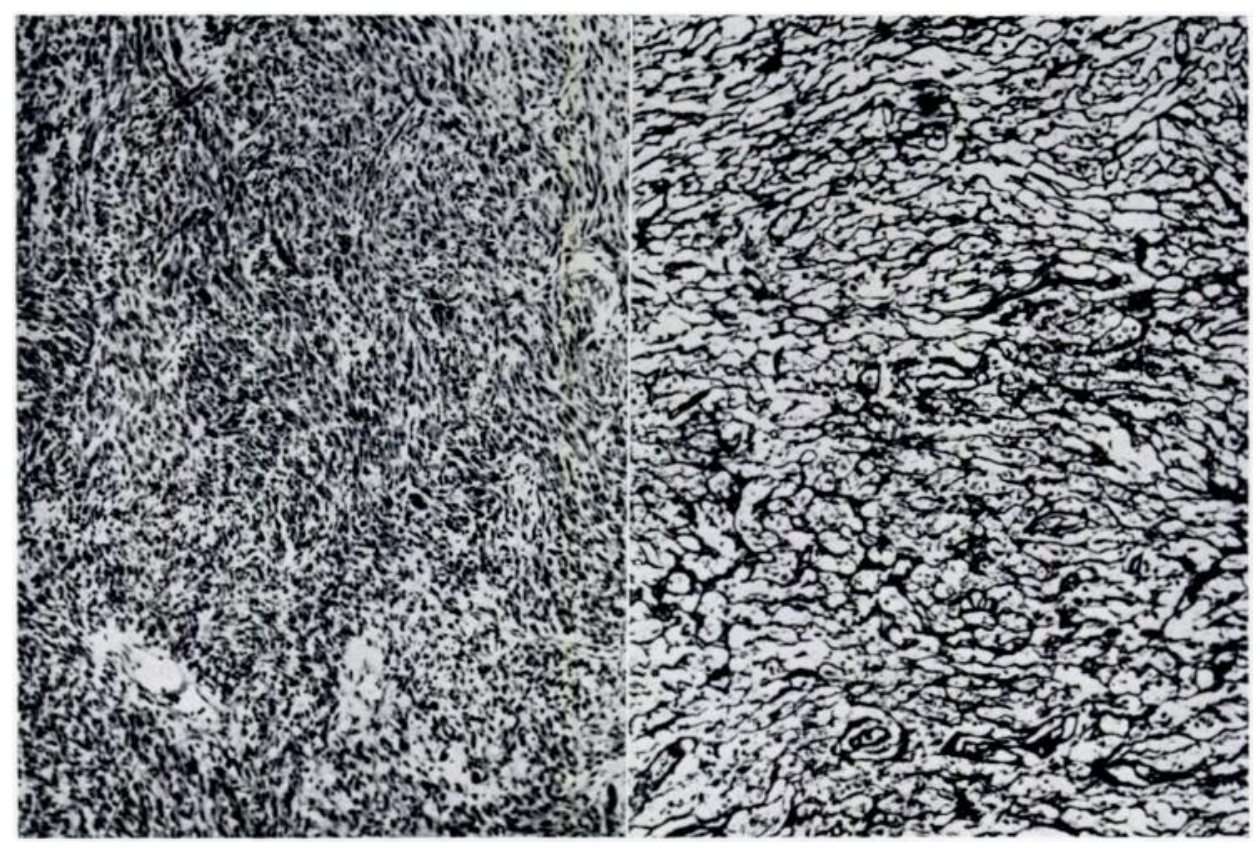

FIG. 4

FIG. 5

Figure 4-Photomicrograph of a representative field showing the cellularity of the tumour with scanty fibre formation $(\mathrm{H}$. and $\mathrm{E} . \times 70)$. Figure 5-Photomicrograph of a silver impregnation preparation to show the rich network of reticulin throughout the whole growth $(\times 120)$.

Progress-After amputation the patient's general condition improved rapidly. Two months later, however, he died suddenly after a short illness, with symptoms suggesting a severe gastro-intestinal haemorrhage. Consent for a post-mortem examination was not obtained.

\section{DISCUSSION}

Whereas carcinoma is a well recognised complication of a chronic bone sinus, the occurrence of a fibrosarcoma is unusual. Gillis and Lee (1951) mentioned that they had seen examples of sarcoma which appeared to have arisen in deeper tissues around scars and foreign bodies. But they did not report any case of sarcoma arising in relation to a chronic bone sinus. Cade (1951) remarked that the occurrence of fibrosarcoma as a sequel to war wounds was well known, but he did not elaborate on this statement or discuss fibrosarcoma occurring in chronic bone sinuses. The comparative rarity of this complication can be judged from the following figures: in approximately 4,000 cases of osteomyelitic sinuses studied at the Mayo Clinic, malignant changes were found in 0.23 per cent, but no sarcomata were reported (McAnally and Dockerty 1949). In one case the tumour was reported as a "grade 3 epithelioma and fibrosarcoma," but a review of other sections revealed that it was actually an epithelioma simulating a fibrosarcoma. Henderson and Swart (1936) reported one case of fibrosarcoma 
arising in connection with a sinus. This tumour arose twenty-two years after the onset of osteomyelitis in the tibia. The limb was amputated and histologically the tumour was thought to be a fibrosarcoma, but four months later the inguinal glands were found to be enlarged and a biopsy of these revealed epidermoid carcinoma. This case, therefore, like that of McAnally and Dockerty, should be classified as a carcinoma, as the original histological interpretation is open to doubt.

Kirshbaum (1949) reported a case of osteomyelitis of the tibia of thirty-one years' standing. An ulcer and a mass developed at the site of the sinus. Amputation was undertaken and there was no recurrence in three years. Microscopy proved the tumour to be a fibrosarcoma. Geschickter (quoted by Kirshbaum 1949) described a similar case, but no details were given.

In the case reported above the clinical diagnosis was of a carcinoma, and the biopsy report of fibrosarcoma was unexpected. It is evident from the histological findings, which are illustrated in the accompanying photomicrographs, that the tumour arose from connective tissue and not from the epidermis.

Fungation of a fibrosarcoma through the skin is uncommon and is virtually seen only after a surgical incision for biopsy or removal. In this case, and in others described in association with sinuses, circumstances exist in which fungation might reasonably be expected.

No conclusion can be drawn from the few cases published as to the relative prognosis between epithelioma and sarcoma. The etiological factors and the natural history of the condition are probably very similar in each.

My thanks are due to Mr H. L.-C. Wood, under whose care the patient was, for his help and advice, and to Dr G. F. M. Hall of the Morbid Anatomy Department, King's College Hospital, for the pathological reports.

\section{REFERENCES}

CADE, Sir S. (1951): Soft Tissue Tumours: Their Natural History and Treatment. Proceedings of the Royal Society of Medicine (Section of Surgery), 44, 19.

Gillis, L., and LeE, S. (1951): Cancer as a Sequel to War Wounds. Journal of Bone and Joint Surgery, 33-B, 167.

Henderson, M. S., and Swart, H. A. (1936): Chronic Osteomyelitis Associated with Malignancy. Journal of Bone and Joint Surgery, 18, 56.

Kirshbaum, J. D. (1949): Fibrosarcoma of the Tibia Following Chronic Osteomyelitis. Journal of Bone and Joint Surgery, 31-A, 413.

McAnally, A. K., and Dockerty, M. B. (1949): Carcinoma Developing in Chronic Draining Cutaneous Sinuses and Fistulas. Surgery, Gynecology and Obstetrics, 88, 87.

Marks, K. L., and Turner, W. L. (1950): Carcinoma Occurring in the Sinuses of Chronic Osteomyelitis. British Journal of Surgery, 38, 209. 